\title{
Legitimación activa y los Tribunales Ambientales (Segundo Tribunal Ambiental)
}

\author{
Comentario de Iván Hunter Ampuero ${ }^{1}$
}

Santiago, tres de marzo de dos mil catorce

Vistos:

$(\ldots)$

\section{LEgitimaCión ACTIVA PARA FORMULAR RECLAMACIONES CONTRA RESOLUCIONES DE LA SMA}

Tercero: Que, como primer argumento de su defensa, el Superintendente señaló que los reclamantes carecen de legitimación activa por cuanto ninguno de ellos acreditó fehacientemente cómo les afectó directamente la Resolución.

Exenta $\mathrm{N}^{\circ} 477$ reclamada, ni cómo esta afectación tendría como causa basal la citada resolución. La SMA distinguió, en su informe presentado en autos el 8 de julio de 2013, entre la legitimación activa en el procedimiento administrativo y la legitimación activa jurisdiccional ante este Tribunal. Agregó que los requisitos exigidos para ser parte activa en un procedimiento de reclamación ante esta Magistratura son más estrictos que aquellos requeridos para hacerse parte en el procedimiento administrativo sancionador ante la SMA, ya que en el primer caso se requiere - para actuar activamente- ser directamente afectado por la resolución, de manera tal que no necesariamente los legitimados del procedimiento administrativo sancionador tendrán la misma calidad ante los Tribunales Ambientales.

Cuarto: Que la legitimación activa para impugnar resoluciones dictadas por la SMA se encuentra regulada en los artículos 56 de la Ley $\mathrm{N}^{\circ} 20.417$ y $18 \mathrm{~N}^{\circ} 3$ de la Ley $\mathrm{N}^{0}$ 20.600. El primer precepto, en su inciso primero, señala: Los afectados que estimen que las resoluciones de la Superintendencia no se ajustan a la ley, reglamentos o demás disposiciones que le corresponda aplicar podrán reclamar de las mismas, dentro del plazo de quince días hábiles, contado desde la notificación, ante el Tribunal Ambiental. Por su parte, la segunda disposición expresa que pueden reclamar en contra de las resoluciones de la SMA las personas naturales y jurídicas directamente afectadas por la resolución de la Superintendencia. De los citados preceptos se puede concluir que la legitimación activa para impugnar

* Profesor de Derecho Procesal, Universidad Austral de Chile. Correo electrónico: ivanhunter@uach.cl 
resoluciones de la SMA está asociada al concepto de "afectado(s)", y como consecuencia de lo señalado en el artículo $18 \mathrm{~N}^{\circ} 3$ de la Ley $\mathrm{N}^{\circ} 20.600$, el afectado por la resolución debe serlo "directamente", es decir, que la afectación surja como consecuencia de lo resuelto en la resolución que se impugna.

Quinto: Que en atención a lo anterior y con el objeto de decidir sobre la legitimación activa de los reclamantes, corresponde a este Tribunal precisar si éstos gozan o no de la calidad de directamente afectados por la Resolución Exenta No 477 de 24 de mayo de 2013, lo que se traduce en determinar la calidad jurídica de los reclamantes en su relación con la citada resolución. Como en todo proceso judicial, la calidad jurídica de legitimado activo normalmente se puede determinar con los antecedentes que surgen de los escritos de las partes y en este caso, además, con los antecedentes que obran en el proceso administrativo sancionatorio que concluyó con la resolución impugnada y los demás procesos administrativos que se encuentran relacionados con los hechos que motivaron las sanciones.

Sexto: Que entre los directamente afectados por una resolución que impone sanciones se debe contar en primer lugar y sin lugar a dudas, al infractor. En el caso de autos, a pesar de la magnitud de la sanción impuesta, éste no reclamó de ella, sino que fueron otras personas -naturales y jurídicas- las que recurrieron contra la resolución sancionatoria requiriendo la imposición de sanciones más severas. La propia SMA parece aceptar que los directamente afectados pueden ser personas distintas del infractor, toda vez que su alegación respecto a la falta de legitimación activa de los reclamantes se basó en la ausencia de prueba de la afectación y no en el hecho de que estos reclamantes no hayan tenido la calidad de infractores en el proceso sancionatorio, a saber: En el caso concreto los reclamantes no se encuentran directamente afectados por la Resolución impugnada, en tanto no han acreditado fehacientemente cómo se encuentran afectados por la Resolución Impugnada y cómo esta afectación tiene como causa basal el actuar de la Superintendencia del Medio Ambiente (Informe de la SMA, fojas 386).

Séptimo: Que tal como lo reconoce la SMA, en el caso de autos algunos de los reclamantes se apersonaron y tuvieron la calidad de interesados en el proceso administrativo sancionador que se llevó ante el ente fiscalizador. Se trata de las Comunidades Indígenas Diaguitas ( $\mathrm{Rol} \mathrm{R} \mathrm{N}^{\circ}$ 7-2013) y las sociedades Agrícola Santa Mónica Limitada y Agrícola Dos Hermanos Limitada (Rol No 8-2013). Por otra parte, también existen reclamantes que no se apersonaron formalmente en el proceso administrativo que culminó con la Resolución Exenta No 477 y que de todas formas impugnaron la citada resolución, como es el caso de los agricultores y regantes de la comuna del río Huasco (Rol N 6-2013).

Octavo: Que este Tribunal se pronunciará primeramente sobre la situación de los reclamantes que tuvieron la calidad de interesados en el proceso administrativo sancionador ante la SMA, comenzando con los denunciantes, las sociedades Agrícola Santa Mónica 
Limitada y Agrícola Dos Hermanos Limitada, continuando luego con las Comunidades Indígenas Diaguitas. Finalmente, esta Magistratura se referirá a la situación de quienes no tuvieron la calidad de interesados en el proceso administrativo sancionador, los agricultores y regantes de la comuna de Alto del Carmen.

Noveno: Que las sociedades Agrícola Santa Mónica Limitada y Agrícola Dos Hermanos Limitada fueron denunciantes en el proceso sancionatorio seguido en contra de la Compañía. Con fecha 8 de febrero de 2013 denunciaron los hechos ante la SMA, denuncia que fue acogida el 12 de febrero de 2013 mediante Resolución Exenta $N^{0}$ 143. En su libelo señalaron ser agricultores del valle del río Huasco, que sus predios se encontraban aguas abajo de la propiedad que opera la Compañía y, que por tanto, sufrían los efectos directos de cualquier clase de contaminación que afecte las aguas del río Chollay y demás afluentes del río Huasco, pues se encuentran dentro del área de influencia del proyecto. En su parte petitoria solicitaron a la SMA que calificara los incumplimientos denunciados como gravísimos y que impusiera a la Compañía determinadas sanciones, ya fuera multa por 10.000 UTA, clausura de faenas o la revocación de la RCA del proyecto.

Décimo: Que el artículo 21 de la LOSMA dispone claramente que, en el caso que un procedimiento administrativo sancionador se iniciare por denuncia, el denunciante tendrá para todos los efectos legales la calidad de interesado en el respectivo procedimiento administrativo. Por aplicación de lo dispuesto en el citado precepto, la SMA mediante Oficio Ordinario $\mathrm{N}^{\circ} 58$ de 27 de marzo de 2013 (considerandos $\mathrm{N}^{\circ}$ 63, 64 y 65) reconoció expresamente la calidad de interesados a las sociedades Agrícola Santa Mónica Limitada y Agrícola Dos Hermanos Limitada. En consecuencia, respecto de estos reclamantes debe entenderse que, por disposición expresa de la LOSMA, se consideran personas cuyos derechos o intereses pueden ser afectados por el proceso administrativo sancionador respectivo y por la Resolución Exenta $N^{\circ} 477$ de la SMA que le puso término e impuso determinadas sanciones al infractor.

Undécimo: Que, al respecto, es necesario tener presente que el artículo 21 de la Ley $\mathrm{N}^{\circ} 19.880$, precisa que interesados en el procedimiento administrativo son quienes: i) lo promuevan como titulares de derechos o intereses individuales o colectivos; ii) los que sin haber iniciado el procedimiento, tengan derechos que puedan resultar afectados por la decisión que en el mismo se adopte; y iii) aquellos cuyos intereses individuales o colectivos, puedan resultar afectados por la resolución y se apersonen en el procedimiento en tanto no haya recaído resolución definitiva. Por lo tanto, en este caso, es la propia ley (art. 21 de la LOSMA) la que ha puesto al denunciante en calidad de interesado en el procedimiento administrativo sancionador, y lo ha hecho "para todos los efectos legales".

Duodécimo: Que de la definición de interesado contenida en el artículo 21 de la Ley $\mathrm{N}^{\circ} 19.880$, se sigue que el denunciante interesado en un procedimiento administrativo sancionador tiene, a lo menos, un derecho o interés que puede ser afectado 
por la resolución que se dictará en el respectivo procedimiento. Es decir, respecto del denunciante es la propia ley la que reconoce una eventual relación de afectación entre su derecho o interés y la resolución absolutoria o sancionatoria que ponga término al proceso sancionatorio, siendo en este contexto y en su calidad de interesado que se le permite hacer valer una pretensión frente a la autoridad administrativa. En este caso, el denunciante interesado solicitó que se calificara la gravedad de las infracciones de la Compañía de una determinada manera y que se le aplicaran determinadas sanciones.

Decimotercero: Que es del todo lógico afirmar que quien fue denunciante interesado -y a quien la propia SMA le reconoció su calidad de tal en el proceso administrativo sancionatorio tenga derecho a reclamar de la resolución que no dio lugar a sus pretensiones. Por lo tanto, en este entendido, se debe concluir que la Resolución Exenta $\mathrm{N}^{\mathrm{o}} 477$ afecta a las sociedades denunciantes en forma directa, por cuanto han sido interesados en el procedimiento administrativo y han hecho valer determinadas pretensiones que no fueron acogidas en la resolución de término que adoptó la SMA. Una interpretación contraria nos llevaría a concluir que, en este caso, quien adquiere la calidad de interesado en el procedimiento administrativo sancionatorio y hace valer una pretensión relativa a la sanción que debe aplicar la administración, no podría reclamar ante este Tribunal a menos que probara fehacientemente su calidad de directamente afectado, más allá de la afectación que implica el no haber obtenido la pretensión que hizo valer ante la autoridad administrativa. En opinión de este Tribunal, el solo hecho de no haber obtenido su pretensión es suficiente afectación para los efectos del artículo 56 de la LOSMA y $18 \mathrm{~N}^{\mathrm{o}} 3$ de la Ley 20.600. Por otra parte, si no se reconoce a los interesados que hayan hecho valer sus pretensiones en un proceso sancionatorio el derecho a reclamar ante este Tribunal, se estaría vulnerando el principio de acceso a la justicia en materia ambiental, consideración sobre la cual el Tribunal volverá más adelante.

Decimocuarto: Que, en conclusión, las sociedades agrícolas fueron denunciantes, tuvieron la calidad de interesados en el proceso administrativo sancionatorio y la resolución de término no impuso las sanciones que ellos consideraban que en derecho correspondía aplicar a la infractora. Debido a lo anterior, han de considerarse perjudicados por la Resolución Exenta No 477 de la SMA, teniendo en consecuencia, la calidad de directamente afectados por dicha resolución, conforme al artículo 56 de la LOSMA y $18 \mathrm{~N}^{\circ} 3$ de la Ley $\mathrm{N}^{\circ} 20.600$.

Decimoquinto: Que, por su parte, el 4 de abril de 2013 las Comunidades Indígenas Diaguitas solicitaron que se les tuviera como interesados en el proceso administrativo sancionador que finalizó con la resolución recurrida. En su solicitud, dichas comunidades argumentaron que cumplían con los requisitos para ser admitidos como interesados conforme a los numerales 2 y 3 del artículo 21 de la Ley $\mathrm{N}^{\circ} 19.880$, por cuanto desarrollan actividades agrícolas y de pastoreo en los territorios donde se lleva a cabo el proyecto, pudiendo ser perjudicados por los efectos ambientales de 
éste. En su parte petitoria, requirieron a la SMA para que acusara y sancionara a la Compañía con la clausura y revocación de la RCA del proyecto, pretensión que no fue acogida por la autoridad administrativa. Por su parte, mediante Resolución Exenta $\mathrm{N}^{\circ} 340$ de 15 de abril de 2013, la SMA acogió la solicitud concediéndoles expresamente el "carácter de interesados en el procedimiento". Una vez reconocida dicha calidad, las Comunidades Indígenas Diaguitas, participaron activamente dentro del proceso sancionatorio, solicitando diligencias y realizando peticiones concretas a la autoridad administrativa.

Decimosexto: Que, en consecuencia, y en atención a lo expresado por este Tribunal en las consideraciones anteriores, las Comunidades Indígenas Diaguitas fueron actores interesados en el proceso administrativo sancionatorio, y al no haber obtenido sus pretensiones en la resolución que puso fin a dicho proceso, sin duda tienen la calidad de directamente afectados por la Resolución Exenta $\mathrm{N}^{\circ} 477$ y gozan de legitimación activa para reclamar ante este Tribunal conforme al artículo 56 de la LOSMA y $18 \mathrm{~N}^{\circ} 3$ de la Ley $\mathrm{N}^{\circ} 20.600$.

Decimoséptimo: Que además, tanto a las sociedades agrícolas denunciantes como a las comunidades diaguitas que se hicieron parte en el proceso administrativo sancionatorio, les asiste otra razón para ser consideradas como "directamente afectados" por la Resolución Exenta N N $^{\circ} 77$. Lo anterior, en atención a su condición de personas que habitan o desarrollan sus actividades dentro del área de influencia del proyecto. Para desarrollar este segundo ámbito por el cual los interesados también se han visto directamente afectados por la resolución reclamada, es necesario tener presente el contexto dentro del cual la SMA ha hecho uso de su potestad sancionadora. En este caso particular, la SMA ha fiscalizado y sancionado a la Compañía infractora por diversos cargos, entre los cuales se encuentran incumplimientos a la RCA del proyecto. La RCA contiene condiciones, normas y medidas para proteger los componentes ambientales y la salud de las personas, componentes que a su vez se vinculan con los derechos e intereses de quienes pueden verse afectados por estar dentro del área de influencia del Proyecto. Por lo tanto, en la medida que en este caso existe una relación entre las condiciones, normas y medidas establecidas en la RCA, las infracciones acusadas, y el desarrollo de la vida y actividades de las personas que habitan o utilizan los recursos hídricos del área de influencia del proyecto, se puede decir que estas personas ostentan la calidad de directamente afectadas por la resolución que pone término al proceso sancionatorio. Esto es así, por cuanto la correcta fiscalización y sanción de las infracciones de las condiciones impuestas por la autoridad sobre el proyecto, favorece a quienes se benefician de los componentes ambientales protegidos por dichas condiciones. De esta manera, si las resoluciones sancionatorias de la SMA no se han ajustado a la ley, los reglamentos o demás disposiciones que le corresponda aplicar, los titulares de los intereses o derechos vinculados a los componentes ambientales y la salud de las personas que se pretendía proteger mediante las normas, condiciones y medidas contempladas en la respectiva RCA, sin duda deben ser considerados como directamente afectados. 
Decimoctavo: Que respecto a los agricultores y regantes de la comuna de Alto del Carmen, estos no fueron -a diferencia de los otros reclamantes- parte interesada en el proceso administrativo sancionador que culminó con la resolución recurrida. Sin embargo, ellos alegan que presentaron una carta de denuncia solicitando que se les reconociera dicha calidad, pero que la SMA no habría proveído ni incorporado el escrito al expediente sancionatorio. Analizada por este Tribunal la citada "carta denuncia" que rola a fojas 1 del expediente de autos, se puede colegir que en dicho documento no se solicitó al Superintendente que se los tuviera como parte interesada en el proceso administrativo sancionatorio, sino que se advierte a la autoridad administrativa de una serie de irregularidades que se habrían suscitado al interior de la Junta de Vigilancia de la Cuenca del Río Huasco y sus Afluentes a la cual dicen pertenecer. A lo anterior, se suma que el comentado escrito no cumple, desde el punto de vista formal, con los requisitos que debe contener una denuncia conforme al artículo 47 de la LOSMA.

En consecuencia, el Superintendente no incurrió en falta por no haberlos considerado interesados en el proceso administrativo sancionador, sin perjuicio de que debió de todas maneras proveer el escrito e incorporarlo al expediente del proceso, omisión que no incidió en la sustanciación del proceso y, conforme al principio de trascendencia, no afecta a la legalidad de la resolución impugnada. Con todo, que la carta no haya tenido el carácter de denuncia, no es óbice para desestimar su contenido con el fin de precisar si los agricultores y regantes de la comuna de Alto del Carmen han sido o no directamente afectados por la Resolución Exenta No 477. En efecto, del documento en cuestión se deducen dos elementos importantes para determinar dicha situación jurídica, los que se tendrán en cuenta para dirimir el asunto.

El primero, que los reclamantes afirman ser miembros de la Junta de Vigilancia de la Cuenca del Río Huasco y sus Afluentes, organización que fue denunciante y a quien la SMA, mediante Oficio Ordinario No 58 de fecha 27 de marzo de 2013, le reconoció el carácter de interesado. El segundo, que manifestaron expresamente ante el Superintendente la pretensión de que se condenara a la Compañía con la revocación de la RCA.

Decimonoveno: Que para determinar si los reclamantes agricultores y regantes de la comuna de Alto del Carmen tienen o no la calidad de directamente afectados por la resolución impugnada, se deben tener presente una serie de consideraciones generales y particulares, muchas de las cuales ya fueron señaladas al confirmar la legitimación activa de los reclamantes que formaron parte del proceso administrativo sancionador y que son las siguientes: i) que las normas, condiciones y medidas establecidas en la RCA tienen por objeto proteger los componentes ambientales y la salud de las personas, componentes que a su vez se vinculan con los derechos e intereses de las personas que habitan o realizan sus actividades en el área de influencia del proyecto; ii) que en el caso que una sanción por incumplimiento de la RCA no se ajuste a la ley, reglamentos o demás disposiciones aplicables, los intereses o derechos de quienes se benefician de los componentes ambientales protegidos por dichas normas, condiciones o medidas se ven afectados puesto que la sanción no habrá cumplido correctamente con sus objetivos; iii) que el incumplimiento de la RCA por la Compañía afecta directamente a 
quienes habitan o realizan actividades dentro del área de influencia del proyecto, ya que los mayores incumplimientos se relacionan con el componente agua, principal elemento de preocupación para cualquier regante que se encuentre dentro del área de influencia; y iv) que dichos incumplimientos dieron origen al proceso administrativo sancionador que terminó con la resolución impugnada.

Vigésimo: Que, de acuerdo a lo señalado en la consideración anterior, es fundamental para determinar si los agricultores y regantes de la comuna de Alto del Carmen son directamente afectados por la resolución impugnada, que éstos hayan acreditado a través de cualquier medio idóneo que efectivamente habitan o realizan actividades dentro del área de influencia del proyecto, más allá de la mera afirmación que en esa dirección realizaran en su escrito de reclamación ante este Tribunal. En este sentido, los reclamantes señalaron ante el Superintendente que ellos formaban parte de la Junta de Vigilancia de la Cuenca del Río Huasco y sus Afluentes, persona jurídica que tiene la calidad de denunciante en el proceso administrativo sancionador y que fue admitida expresamente como parte interesada por la SMA mediante Oficio Ordinario $\mathrm{N}^{\circ} 58$ de fecha 27 de marzo de 2013, y que no impugnó la resolución reclamada en autos. Por lo tanto, de ser efectivo que forman parte de la citada Junta de Vigilancia, se derivarían dos importantes consecuencias. Primero, se acreditaría que estos reclamantes habitan o realizan actividades en el área de influencia del proyecto, criterio fundamental para determinar su calidad de "directamente afectado", tal como ya se señaló en la consideración décimo séptima de esta sentencia. Segundo, en cierta medida, su situación sería asimilable a los reclamantes que fueron parte interesada en el proceso administrativo sancionador, ya que pertenecerían a una organización que fue denunciante y considerada como interesada en el proceso administrativo sancionatorio que culminó con la resolución impugnada.

Vigésimo primero: Que este Tribunal solicitó, el 27 de noviembre de 2013 y como medida para mejor resolver, que la parte reclamante acompañara la documentación necesaria que acredite que los regantes de la comuna de Alto del Carmen individualizados en la reclamación formaban parte de la Junta de Vigilancia de la Cuenca del Río Huasco y sus Afluentes.

Dando cumplimiento a la medida solicitada por este Tribunal, el abogado de la parte reclamante acompañó los certificados que acreditaron la calidad de regantes y miembros de la citada Junta de Vigilancia de sólo nueve de los 43 reclamantes, a saber: Nolberto Huanchicay Villegas, Feliz Guerrero Cortes, Fernando del Carmen Vivanco, Horacio Gaytan Arcos, Paulo Herrera Vallejos, Clementina Contreras Carmona, Nibaldo Arcos Ardiles, Eluicura Carmona Calderón y Pascual Olivares Iriarte.

En cuanto a los demás reclamantes, no se acompañó durante el proceso seguido ante este Tribunal ningún antecedente que pudiese avalar que efectivamente fuesen agricultores o regantes de la comuna de Alto del Carmen ni miembros de la citada Junta de Vigilancia, más allá de la mera afirmación en ese sentido contenida en el escrito de reclamación presentado ante este Tribunal. 
Vigésimo segundo: Que, en consecuencia, este Tribunal estima que de acuerdo a las consideraciones señaladas precedentemente, sólo pueden ser tenidos como directamente afectados por la Resolución Exenta No 477 quienes pudieron acreditar su calidad de regantes de la comuna de Alto del Carmen y de miembros de la Junta de Vigilancia de la Cuenca del Río Huasco y sus Afluentes. Se trata, en definitiva, de personas que realizan sus actividades dentro del área de influencia del proyecto y cuyos derechos e intereses se encuentran vinculados a los componentes ambientales protegidos por el contenido de la RCA.

Se suma a lo anterior que la RCA fue incumplida por la Compañía justamente en relación con el recurso más importante para los regantes, el agua, y que la imposición de las sanciones por parte de la SMA les afecta directamente en sus derechos o intereses vinculados al citado componente ambiental que la RCA ha pretendido proteger. Todo lo anterior hace concluir a este Tribunal que sólo los nueve regantes de la comuna de Alto del Carmen individualizados en la consideración anterior, son "directamente afectados" por la Resolución Exenta No 477 y como consecuencia de ello, están, al igual que los otros reclamantes de autos, legitimados activamente para reclamar de dicha resolución ante este Tribunal.

Vigésimo tercero: Que este Tribunal, al reconocer la legitimación activa de las sociedades Agrícola Santa Mónica Limitada y Agrícola Dos Hermanos Limitada, de las Comunidades Indígenas Diaguitas y de los nueve regantes de la comuna de Alto del Carmen ya individualizados, para interponer reclamación judicial contra la Resolución Exenta N 477 del Superintendente del Medio Ambiente, ha sido coherente con el principio de acceso a la justicia ambiental. En efecto, cabe señalar que a diferencia de otras materias jurídicas, los problemas ambientales han expandido, por su misma naturaleza, las fronteras de lo que tradicionalmente se ha entendido por afectado o directamente afectado, llegando en algunas legislaciones incluso a considerar la acción pública cuando se encuentran envueltos conflictos socio ambientales. La legislación nacional, sin reconocer expresamente las denominadas acciones de clase o acción pública en materia ambiental, ha reconocido un ámbito de afectación de derechos e intereses amplio, como lo demuestra la noción de "área de influencia de un proyecto" descrita más arriba. La legitimación activa está relacionada con la afectación de intereses y derecho. Y recordemos que la Ley $\mathrm{N}^{\circ} 20.600$ en su artículo 18 dispone que "[...] las personas naturales o jurídicas que se señalan, podrán intervenir como partes en los asuntos de competencia de los Tribunales Ambientales [...], para luego reiterar en el numeral 3), que respecto de las resoluciones de la SMA podrán reclamar "las personas naturales o jurídicas directamente afectadas". Lo anterior es particularmente importante, pues no solo las personas naturales que habitan o realizan alguna actividad en el área de influencia tienen el denominado locus standi, sino que también las sociedades comerciales agrícolas que actuaron como denunciantes en la presente reclamación.

Vigésimo cuarto: Que esta expansión de la legitimación activa ante problemas ambientales, ha sido por de pronto la respuesta correcta a aquellas garantías procesales contenidas 
en el Principio 10 de la Declaración de Río sobre el Medio Ambiente y el Desarrollo de 1992, donde los Estados, incluido Chile, acordaron que Deberá proporcionarse acceso efectivo a los procedimientos judiciales y administrativos, entre éstos el resarcimiento de daños y los recursos pertinentes, garantías que fueron desarrolladas en el Programa 21 de Río, que exhorta a los gobiernos y legisladores a establecer procedimientos judiciales y administrativos de indemnización y reparaciones (...), y que deberian propiciar el acceso de personas, grupos y organizaciones que tengan un interés jurídico reconocido (Programa 21, Capítulo 8, punto 8.18).

Mientras tanto, en el concierto internacional, particularmente en el ámbito europeo, se ha materializado con contundencia esta garantía, como da cuenta la Convención de Aarbus sobre acceso a la información, participación pública en la toma de decisiones y acceso a la justicia en temas medio ambientales, suscrita en Dinamarca en 1998, donde se prescribe que cualquiera que tenga un "interés suficiente" o invoque "la lesión de un derecho", podrá interponer recurso ante un órgano judicial u otro órgano independiente e imparcial establecido por la ley para impugnar la legalidad, en cuanto al fondo y en cuanto al procedimiento, de cualquier decisión, o cualquier acción u omisión que se encuentre dentro del ámbito de los derechos consagrados en dicho Convenio (artículo 9 No 2 de la Convención).

Vigésimo quinto: Que, por su parte, esta ampliación de la noción de legitimado activo ante decisiones administrativas garantiza también que no se incurra en "discriminación ambiental". De acuerdo con esta noción, es posible distinguir varias situaciones de discriminación o injusticia ambiental, como cuando - por ejemplo- una parte de la población carga con la mayoría de los pasivos ambientales mientras otra con los beneficios. Sin embargo, el caso de autos apunta más bien a que existiría discriminación si el Tribunal incluyera dentro de los legitimados activos sólo a los denunciantes, o a estos y a los que hayan sido tenidos como interesados en el procedimiento administrativo, y no a quienes, sin ser interesados en dicho procedimiento, habiten o realicen sus actividades en el área de influencia del proyecto, o vice versa. Por último, es importante tener presente, que si bien el concepto de "discriminación ambiental" no se encuentra consagrado expresamente en nuestro ordenamiento jurídico, éste se deriva del principio de igualdad y de la garantía de no discriminación, consagrados en el numeral $2^{\circ}$ del artículo 19 de la Constitución Política de la República.

Vigésimo sexto: Que, por último, cabe recordar aquí, en atención a la ampliación que ha ganado con el tiempo la legitimación activa tanto en temas ambientales como en otros problemas de carácter difuso, el brillante trabajo dirigido por Mauro Cappelletti en 1975 sobre el acceso a la justicia, y que dice: Estamos asistiendo a la lenta pero segura declinación de una concepción individualista del proceso y la justicia. Todos los principios, los conceptos, las estructuras que estaban radicadas en esa concepción, aparecen cada día más insuficientes para dar una aceptable respuesta al problema de asegurar la tutela necesaria a los nuevos intereses difusos y de grupo, que han llegado a ser vitales para las sociedades modernas (Proyecto"Florencia para el Acceso a la Justicia", en Access to Justice: a World Survey (Cappelletti y Garth, compiladores, cuatro volúmenes, Editoriales Sijthoff (Leyden y Boston) y Giuffrè (Milán), 1975). 


\section{Comentario}

Como es de esperar la jurisprudencia de los Tribunales Ambientales irá fijando paulatinamente -y ojalá en términos consistentes y coherentes-, el sentido de algunas reglas de la ley 20.600 (en adelante LTA). La sentencia de Pascua Lama dictada por el Segundo Tribunal Ambiental (TA), en causa Rol N ${ }^{\circ}$ 6-2014, es un buen ejemplo, no sólo porque en este fallo se centra la mirada de muchos actores políticos y sociales relacionados con la cuestión ambiental, sino además porque se han fijado algunos estándares de acceso a los tribunales ambientales. Y es que la relación entre procedimiento administrativo y proceso judicial es un problema que sobrepasa a la simple pregunta acerca de la deferencia de las decisiones entre una u otra. Mi interés es concentrarme en algunas cuestiones relacionadas con la legitimación activa.

El mundo académico planteó una preocupación inicial por el sentido y alcance del Art. 18 LTA respecto de la legitimación para acceder al tribunal ambiental (mal llamado "partes" por la ley). Se consideraba que algunos de los numerales de esta disposición eran muy restrictivos y difícilmente realizables en la práctica; por otro lado, siendo la legitimación un criterio de selección de intereses jurídicamente relevantes, la opción legislativa de privilegiar el acceso casi exclusivamente a los titulares de proyectos y actividades empresariales era al menos criticable. Ambas críticas están en lo correcto aunque deben ser morigeradas a partir de esta sentencia.

En este fallo el TA ha interpretado la expresión "directamente afectado" del Art. 56 LOSMA, y $18 \mathrm{~N}^{\circ} 3$ LTA en un sentido amplio. Bajo esta rúbrica se encontrarían: a) el denunciado o presunto infractor; b) el denunciante; c) los interesados en el procedimiento administrativo sancionador, pudiendo distinguirse a los que comparecieron al procedimiento, y los que siendo interesados, no lo hicieron. El esfuerzo argumentativo de la sentencia es notable y su precedente también: ampliar el acceso a los tribunales ambientales, posibilitando que incluso aquellos que no han tenido participación en el procedimiento administrativo puedan impugnar la resolución de la Superintendencia cuando acrediten estar afectados por la misma ${ }^{1}$. También se lee-aunque entrelíneas-que la declaración de la calidad de "interesado" que hace la Administración en el seno de un procedimiento administrativo es suficiente para legitimar el acceso a la tutela judicial y que esa calidad no puede ser cuestionada por la jurisdicción (considerando decimosexto).

Ahora bien, la sentencia en cuestión también deja algunos espacios para la reflexión. El primero es la corrección de la identificación entre denunciante, interesado y afectado. Es cierto que el Art. 21 LOSMA le da al denunciante el carácter de interesado en el procedimiento administrativo. Pero esa condición, cuando se hace referencia a la impugnación

${ }^{1}$ Jorge Bermúdez ya había insinuado esta posibilidad en el 2013, en su artículo "Administración y Jurisdicción Ambiental: la institucionalidad ambiental frente al nuevo contencioso administrativo", en La nulidad de los actos administrativos en el derecho chileno. IX Jornadas de Derecho Administrativo, Ferrada, Juan Carlos (Coords.), Thomson ReutersLegalPublishing, 2013, p. 502. 
de la resolución puede en ocasiones separarse de la de afectado. A mi entender, esta norma - usando una expresión de Schauer ${ }^{2}$, es una generalización probabilística implícitamente justificada. En efecto, en la generalidad o la mayoría de los casos quien actúa como denunciante, colaborando con la función administrativa, tiene un interés privado que se tutela por medio de la satisfacción del interés público. Sin embargo, es perfectamente posible que en casos particulares la conexión entre el objeto de la regla (denunciante como interesado en el procedimiento administrativo) y su justificación (denunciante tutela su interés por medio de la satisfacción del interés público) esté ausente ${ }^{3}$. De ahí entonces que el TA deba precisar, como bien lo hace en este fallo, si la regularización justificativa de la regla que le otorga al denunciante la calidad de interesado, se encuentra presente (o ausente) en el caso concreto. De lo contrario, estaríamos admitiendo que el simple interés de colaboración con la Administración o del cumplimiento de la ley es suficiente para provocar la actuación jurisdiccional.

La principal consecuencia de esta interpretación es que el Art. 21 LOSMA sería una regla de prueba y de justificación del interés respecto del denunciante. Quien figura como denunciante no deberá ni probar ni justificar su interés en el procedimiento administrativo sancionatorio. Por el contrario, quien quiera desconocerlo correrá con la carga de justificación y prueba ${ }^{4}$.

En segundo lugar, y el otro aspecto que la sentencia deja en el aire, es si posible concebir algún otro criterio, distinto al de área de influencia, para estimar que ha existido afectación de intereses y justificar la legitimación. No creo que la intención del fallo sea cerrar la puerta a otros criterios, pero el interesado deberá acreditar el beneficio o utilidad que le produzca la aplicación de una sanción al titular de un proyecto o actividad.

${ }^{2}$ Schauer, Frederick, Las reglas en juego. Un examen filosófico de la toma de decisiones basadas en reglas en el derecho y en la vida cotidiana. Marcial Pons, Madrid, 2004, p. 90.

${ }^{3}$ Ibíd., pp. 90 y 91. Las normas, más allá de sus justificaciones subyacentes, gozan de una autonomía semántica que las hace aplicables sin necesidad de encontrar sus justificaciones. Sin embargo, como explica Rodenas, “esta autonomía semántica de las formulaciones normativas puede dar lugar a que en algunos casos se produzca un desajuste entre aquello que la formulación normativa nos exige y lo que la justificación subyacente a la misma requiere". Rodenas, A, "En la penumbra: Indeterminación, derrotabilidad y aplicación judicial de las normas", en Doxa, $\mathrm{N}^{\circ} 24$, pp. 20 y 21 . Con todo, una interpretación como esta puede reconducirse a lo que se entiende por interpretación restrictiva desde que es posible al momento de interpretar la norma jurídica introducir una distinción que el autor de la norma no ha efectuado, con la finalidad de reducir su ámbito de aplicación. Vid., Moreso, J., y Vilajosana, J., Introducción a la teoría del derecho, Marcial Pons, Madrid, 2004, p. 208.

${ }^{4}$ Esta interpretación es además coherente con lo sustentado por la doctrina en el derecho comparado, donde el denunciante no adquiere, por esa sola circunstancia, la calidad de interesado en el procedimiento, debiendo, en consecuencia, justificar la existencia de un derecho e interés afectado. Vid., Nieto, Alejandro, Derecho administrativo sancionador, Editorial Tecnos, Madrid, 2012, p. 113. 
Por último, y en otra arista, la aceptación de la legitimación de los interesados que no han participado en el procedimiento administrativo abre la disputa acerca desde cuándo debe computarse el plazo para impugnar. La experiencia jurisprudencial en otros contenciosos, como en el reclamo de ilegalidad municipal, no ha sido muy auspiciosa, postulándose tres tesis diferentes: desde que se dicta el acto; desde que se toma conocimiento efectivo del acto; o desde que no pudo menos que tomarse conocimiento de éste.

La sentencia en comento constituye un notable avance en la materia, y seguramente, será un hilo que seguirán los demás tribunales ambientales. 